renouveler la culture, assurant ainsi la continuité et le renouvellement vers les générations futures. Aussi surprenant que cela puisse être, la lecture de ce livre nous amène inconsciemment à réfléchir sur notre cheminement personnel et à nous situer entre ce que nous avons accompli et ce que nous désirons accomplir.

Or, bien que cet ouvrage s'adresse aux professionnels œuvrant dans les organisations, il

\section{Jason Fried et David Heinemer Hansson, Réinventer le travail, Éditions Transcontinental, 2010, 230 p.}

Une approche iconoclaste de la gestion: Réinventer le travail bouscule les idées reçues. La planification relève davantage de l'art divinatoire que de la gestion, travailler plus ne conduit pas à améliorer les résultats, il faut s'inspirer des vendeurs de drogues pour déployer sa force de vente et sa stratégie commerciale. Il s'agit là de quelques propositions de Fried et Hansson pour réinventer le travail dans les milieux organisationnels.

En matière de gestion des ressources humaines, ils nous proposent de réduire l'embauche, de ne pas tenir compte des CV, de l'expérience et des diplômes. Il est préférable d'opter pour des personnes autonomes, qui écrivent bien. En bref, il faut porter une plus grande attention à la lettre de présentation qu'aux $\mathrm{CV}$, invérifiables et souvent truffés d'exagérations.

Pour le marketing, les auteurs affirment qu'il est préférable d'attirer la clientèle plutôt que de la traquer: il faut se rendre intéressant. Plutôt que vendre un ingrédient, mieux vaut publier des recettes. Selon eux, les communiqués de presse sont des pourriels : les clients s'efforcent d'en maîtriser le flot plutôt qu'en prendre réellement connaissance. Donnez votre produit! Si votre produit est bon, les clients reviendront avec de l'argent: utilisez essai gratuit, échantillon ou démonstrateur pour faire connaître et aimer ce que vous offrez. Chaque utilisation du produit est du marketing. nous amène subtilement à porter un regard sur soi et à nous projeter vers un but plus grand que soi, c'est-à-dire, le désir de créer à notre tour la générativité.

\section{Émélie Demers, M.A., Conseillère d'orientation organisationnelle Chaire de recherche sur l'intégration profession- nelle et l'environnement psychosocial de travail Université Laval}

En ce qui concerne la productivité, les auteurs suggèrent de réduire le nombre de réunions, de prendre les petites décisions, de dormir davantage, de ne pas prendre de notes et d'autres moyens pour consacrer davantage de temps aux choses importantes. Pour ces dernières, l'excuse du manque de temps ne tient pas: si on peut passer 20 heures par semaine devant la télévision, on a amplement de temps pour démarrer une entreprise, se tenir en forme et faire beaucoup d'autres choses.

Les idées ne manquent pas dans ce court livre très rafraichissant. Bien que les explications soient relativement convaincantes, elles sont brèves et peu documentées. Les thèmes tiennent sur une page. Les présentations permettent d'une part de déboulonner les activités classiques du management et de proposer des alternatives à contre-courant et originales : apprendre de ses succès plutôt que de ses erreurs, improviser pour plus de mouvement et de flexibilité, rester petit, agir, abolir les messages pour faire patienter les clients au téléphone, ne pas emprunter d'argent, réduire le nombre d'employés, les plans et contrats à long terme, les jeux politiques à l'interne, les réunions, nos ambitions, par exemple.

Les observations et recommandations faites dans Réinventer le travail sont basées en grande partie sur le lancement et la gestion de 37 signals, entreprise des auteurs spécialisée dans les logiciels en gestion de projet, et de gestion des contacts et relations avec la clientèle

Les thèses de l'ouvrage rendent-elles obsolètes les classiques du management? Ça reste à démontrer mais les propositions sont autant 
séduisantes que déstabilisantes. Réussir en réduisant ou abolir l'idée de croissance, les réunions, la préparation de budget, les conseils d'administration, la publicité, la représentation commerciale et ne pas tenir compte de la «dure réalité » constitue selon les auteurs une nouvelle recette du succès. Qui sera l'audacieux entrepreneur qui osera s'aventurer dans cette direction?

Ce livre a la grande qualité de sortir des sentiers battus et de proposer des solutions concrètes. Ces moyens expérimentés dans l'entreprise des auteurs obtiendraient-ils autant de succès dans d'autres contextes ou avec des produits différents? Peut-être pas. Ils valent certainement la peine d'être expérimentés.

Stéphane Aubin Professeur, Université du Québec à Chicoutimi 


\section{Doctorat en \\ développement régional}

\section{ABORDER LE DÉVELOPPEMENT TERRITORIAL SOUS UN ANGLE ORIGINAL?}

Dans ce programme unique en son genre au Québec, l'analyse de l'espace, de son aménagement, sa gestion et son développement socioculturel et économique est intégrée à celle des dynamiques territoriales. Les enjeux tant environnementaux qu'économiques, politiques, sociaux ou culturels auxquels sont confrontés les acteurs territoriaux y sont analysés dans une perspective interdisciplinaire.

\section{POURSUIVRE SES ÉTUDES}

\section{DANS UN CADRE ORIGINAL?}

Le programme est offert conjointement par I'UQAC et l'UQAR qui ont une longue expérience dans la recherche et l'intervention en développement local et régional. Les deux universités misent sur des rencontres en face à face ainsi que sur un usage créatif des plus récentes technologies de la communication pour dispenser l'enseignement et fournir l'encadrement requis par le proramme.

Date limite demande d'admission : $1^{\mathrm{er}}$ mars de chaque année

\section{UQAC}

Université du Québec à Chicoutimi Jean-François Moreau

Téléphone : 418 545-5011, poste 4580

Courriel : pcs_developpement-regional@uqac.ca www.uqac.ca/registr/programmes/3770.html
Université du Québec à Rimouski

Université du Québec à Rimouski Johanne Boisjoly

Téléphone : 418 724-1648, poste 1687

Courriel : druqar@uqar.qc.ca

wer.uqar.qc.ca 